\title{
Spectral Control of an X-Ray $L$-Edge Transition via a Thin-Film Cavity
}

\author{
Johann Haber, ${ }^{1, *}$ Jakob Gollwitzer, ${ }^{1}$ Sonia Francoual, ${ }^{1}$ Martin Tolkiehn, ${ }^{1}$ Jörg Strempfer,,${ }^{1, \dagger}$ and Ralf Röhlsberger ${ }^{1,2}$ \\ ${ }^{1}$ Deutsches Elektronen-Synchrotron DESY, Notkestraße 85, 22607 Hamburg, Germany \\ ${ }^{2}$ The Hamburg Center for Ultrafast Imaging, Luruper Chaussee 149, 22761 Hamburg, Germany
}

(Received 9 October 2018; revised manuscript received 13 February 2019; published 29 March 2019)

\begin{abstract}
By embedding a thin layer of tantalum in an x-ray cavity, we observe a change in the spectral characteristics of an inner-shell transition of the metal. The interaction between the cavity mode vacuum and the $L_{\mathrm{III}}$-edge transition is enhanced, permitting the observation of the collective Lamb shift, superradiance, and a Fano-like cavity-resonance interference effect. This experiment demonstrates the feasibility of cavity quantum electrodynamics with electronic resonances in the x-ray range with applications to manipulating and probing the electronic structure of condensed matter with high-resolution $\mathrm{x}$-ray spectroscopy in an $\mathrm{x}$-ray cavity setting.
\end{abstract}

DOI: 10.1103/PhysRevLett.122.123608

Manipulating the spectral characteristics of matter by embedding it in tailored environments such as cavities or photonic crystals is a crucial tool for modern quantum optics. Beginning with gaseous atomic ensembles and single atoms [1-4], this field, known as cavity quantum electrodynamics (CQED) [5,6], has proven to be an indispensable tool, yielding both practical applications and fundamental insights about matter and its interaction with light. Developments arising from it, such as nonclassical states of light, are important in refining modern experimental capabilities, for instance, in gravitational wave detection and imaging of biological structures [7,8]. CQED-related effects and applications have been demonstrated across a wide range of different energies and resonant systems, from microwaves to visible light. In higher photon energy regimes, however, progress has been slow. $\mathrm{X}$ rays in particular are a preeminent experimental tool for physics and materials science, probing the properties of solids and molecules and delivering information on spatial and electronic structure, dispersion relations of quasiparticles, and chemical properties. Many of these techniques stand to gain from a greater experimental control over the properties of the incoming light, be it the spectral composition, noise or temporal structure afforded by cavity quantum electrodynamics. CQED in the $\mathrm{x}$-ray range could also be used to probe and manipulate many-body interactions within the resonant matter in a more direct way, by placing it into the cavity, which is tuned to certain electronic transitions of the specimen, as suggested for visible light [9-13]. Extending CQED into the X-ray range is therefore highly desirable. Recent years have seen some success in this area, leading to phenomena such as the collective Lamb shift [14], electromagnetically induced transparency [15], spontaneously generated coherences [16], strong coupling [17,18], Fano resonances [19], and slow light [20] being observed. However, all these experiments made use of ${ }^{57} \mathrm{Fe}$, an iron isotope with a nuclear resonance at $14.4 \mathrm{keV}$. While the interaction with nuclear resonances in a cavity QED setting enables fundamental studies and precision metrology of the light-matter interaction, it precludes coupling to electronic degrees of freedom for an active control of the corresponding transitions. In this Letter, we successfully demonstrate that X-ray cavity QED is a viable technique even when using atomic shell resonances in the $\mathrm{x}$-ray range. This drastically enlarges the number of possible applications, permitting the examination of a wide range of different elements and materials. It also extends the spectrum of $\mathrm{X}$-ray wavelengths which may be manipulated with cavity QED techniques, and may enable combining the nascent fields of $\mathrm{x}$-ray CQED and x-ray nonlinear optics [21].

As a model system, we use the electric dipole transition of the $L_{\text {III }}$ edge of tantalum, which has an energy of $9881 \mathrm{eV}$ and a linewidth of approximately $5 \mathrm{eV}$ [22]. It is often referred to as one of the so-called "white lines" of x-ray absorption spectroscopy, and is the result of a transition from the $2 p$ shells to the $5 d$ bands which have a high density of states at the Fermi level [23]. The very large bandwidth has drawbacks as well as advantages. On the one hand, many possible interesting experiments involving temporal control and manipulation, such as the creation of x-ray frequency combs [24] and spectral profile manipulation [25], cannot be performed with these electronic X-ray transitions, since they require temporal control not available at the corresponding short timescales. On the other hand, the large bandwidth means that the brilliance of current $\mathrm{x}$-ray sources may permit multiphoton and nonlinear experiments in the near future. It is also easily generalizable to a number of inner-shell electronic transitions in many elements, extending the number of $\mathrm{x}$-ray resonances available to $\mathrm{x}$-ray CQED beyond the nuclear resonances presently dominant. 
The experiment was performed at the P09 beam line of the PETRA III synchrotron radiation source at DESY, Hamburg, which has a monochromator with a relative bandwidth of $(\Delta E) / E=1.3 \times 10^{-5}$ using $\operatorname{Si}(111)$ crystals. A sketch of the experimental setup is shown in Fig. 1. A thin-film cavity consisting of the layer system $15.4 \mathrm{~nm}$ $\mathrm{Pt} / 12.3 \mathrm{~nm} \mathrm{C} / 2.5 \mathrm{~nm} \mathrm{Ta} / 11 \mathrm{~nm} \mathrm{C/2} \mathrm{nm}$ Pt sputter deposited on a silicon substrate is placed on a goniometer head on a six-circle diffractometer. We used two detectors: an avalanche photodiode (APD) measuring the angle-resolved reflectivity of the sample and a Vortex energy dispersive detector probing part of the fluorescence emitted by the tantalum into $4 \pi$. The fluorescence detector has an energy resolution of about $130 \mathrm{eV}$. In order to only measure the tantalum fluorescence, we scanned the monochromator energy across the Ta $L_{\mathrm{III}}$ absorption edge in order to identify the Vortex detector channels corresponding to the Ta emission line; in the range where the number of

(a)
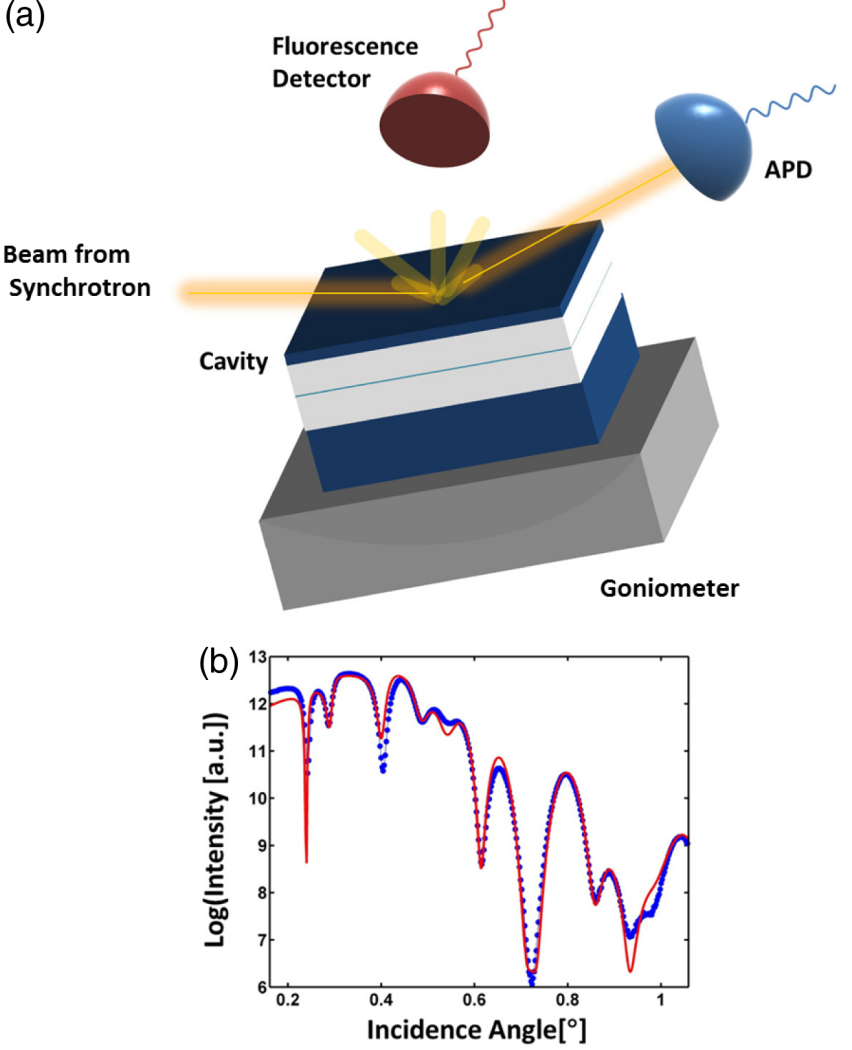

FIG. 1. (a) Experimental setup. The multilayer cavity containing a thin tantalum layer is placed on a goniometer and illuminated at grazing incidence angles by $\mathrm{x}$ rays from $\mathrm{a}$ synchrotron tuned to the Ta $L_{\mathrm{III}}$ edge energy of $9881 \mathrm{eV}$. An avalanche photodiode (APD) is positioned to collect the reflected radiation. Another detector is placed to the side of the cavity to measure a part of the light emitted into $4 \pi$, which is largely fluorescence radiation. (b) The reflectivity curve of the sample, detuned by $70 \mathrm{eV}$ from the edge in order to omit resonant effects. The dips indicate standing wave modes, with the first order mode being the first sharp dip on the left. detected photons grew rapidly at resonance we defined a region of interest (ROI) and in the following summed up all photons in that ROI to exclude photons not stemming from the Ta fluorescence. We introduced a $700-\mu$ m aluminium foil into the beam downstream of the cavity in order not to overload the APD, as well as a stack of 20 compound refractive lenses to focus the beam, as the grazing incidence geometry otherwise would result in a large portion of the beam overpassing the sample. A basic sketch of the setup is shown in Fig. 1(a). First, we performed a simple offresonance $\Theta-2 \Theta$ scan in order to localize the dips in the reflectivity curve which mark the excitation of cavity modes, shown in Fig. 1(b). For a given angle of incidence, the monochromator was scanned between 9800 and $10000 \mathrm{eV}$, a range which encompasses the $9881-\mathrm{eV}$ resonance energy of the $L_{\text {III }}$ transition. We repeated this for a wide range of angles around the first cavity mode, located at $\approx 0.242^{\circ}$. As described above, for each monochromator position and angular setting of the cavity, we recorded all photons detected by the APD, and those in the ROI for the fluorescence detector. The results are shown in Figs. 2 and 3, along with simulations largely confirming the results.

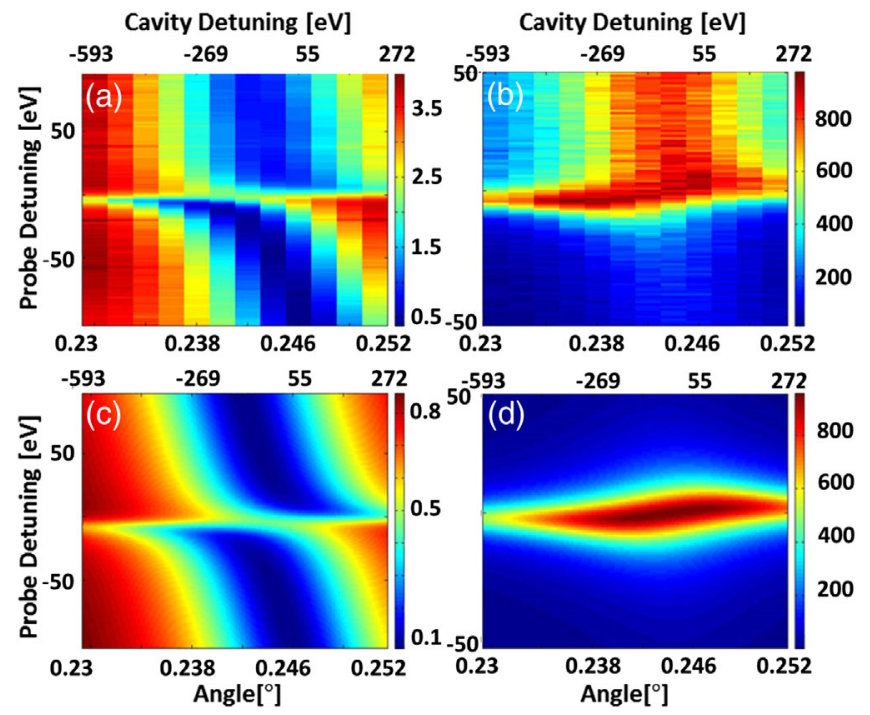

FIG. 2. Spectroscopic signals of the cavity. The intensity is encoded in the color map. The $x$ axis shows the cavity detuning expressed in terms of both angle (bottom axis) and energy (top axis), and the $y$ axis shows the detuning of the monochromator from the transition energy in eV. (a) Reflectivity of the sample. There is an anticrossinglike behavior which is not entirely resolved; rather, the two branches of the dispersion relation interfere, giving Fano-like effects. (b) The fluorescence signal. As the cavity is tuned through the transition line, the latter undergoes some changes; it is slightly shifted by up to $2.5 \mathrm{eV}$ and its spectral width is enhanced to up to $10 \mathrm{eV}$. These effects are the collective Lamb shift and the superradiant decay enhancement, respectively. The large signal at positive monochromator detunings is the fluorescence of the steplike electronic continuum. Panels (c) and (d) show simulations of (a) and (b), respectively, without taking the effects of the electronic continuum into account. 

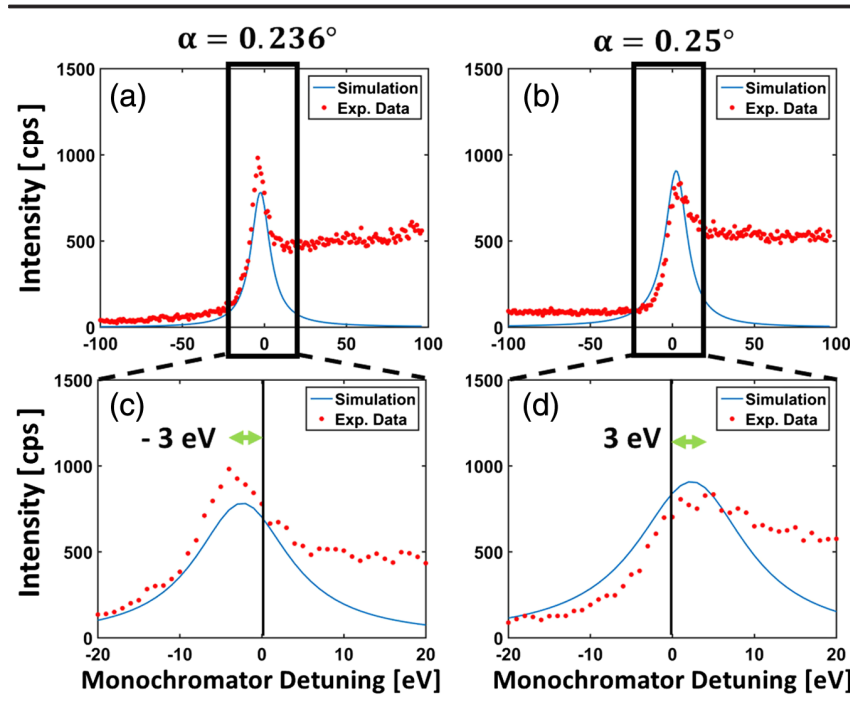

modes absorbing light, we here see only a narrow peak. This is quite expected: almost only light absorbed by the tantalum is scattered into $4 \pi$, and therefore a signal is only observed when the incoming radiation has more or less the precise energy of the tantalum $L_{\mathrm{III}}$ transition. A qualitatively new phenomenon, however, is the dispersion of this peak. It is clearly visible that while it is exactly at $9881 \mathrm{eV}$ at the cavity mode center, it veers off this value when the cavity is slightly detuned, only to return to it at larger detuning. The shifts are as large as $2.5 \mathrm{eV}$ at particular points, as shown in Fig. 3. Furthermore, when the cavity is not detuned at all, the linewidth is slightly broadened. These phenomena are well known as the collective Lamb shift and single-photon superradiantly enhanced decay, respectively $[14,27]$. The superradiance is a phenomenon resulting from the collective excitation of the sample; an ensemble of emitters that is excited in phase will emit its photons much faster [28]. The collective Lamb shift is a phenomenon that has been the focus of intense theoretical [27,29-39] and experimental [14,40-43] attention. It stems from a dipole-dipole interaction induced by the electromagnetic vacuum, which in our scenario is enhanced by the $\mathrm{x}$-ray cavity, enabling the observation of this weak effect. The cavity vacuum induces virtual photon transitions from one atom to the next. These lead to a slight shift of the collective excitation state- the collective Lamb shift.

The simulations do not take into account the presence of the Heaviside-like absorption step due to the electronic continuum which begins right after the onset of the white line. This explains the grave discrepancies at positive energy detunings which are clearly visible in both spectra on Fig. 3 or Figs. 2(b) and 2(c).

The dispersion of the dips in Fig. 2(a) and of the fluorescence line in Fig. 2(b), as well as their widths, are well described by the model given in Ref. [27]. While the latter is formally devised to describe the interaction of $\mathrm{x}$ rays with nuclear excited states, it serves just as well to describe that with the electronic shell excited states. It has been used extensively $[18,19]$, and therefore only the principal results are recalled here. The reflectivity is given by

$$
\begin{aligned}
R= & -1+\frac{2 \kappa_{R}}{\kappa+i \Delta_{c}} \\
& -\frac{i \sqrt{2 \kappa_{R}}}{a_{\mathrm{in}}\left(\kappa+i \Delta_{c}\right)} \frac{|g|^{2} N \Omega}{\Delta+i \gamma+|g|^{2} N\left(i \xi_{s}-\delta_{\mathrm{LS}}\right)},
\end{aligned}
$$

where $\kappa$ is the decay constant of the cavity and $\gamma$ that of the resonant atoms, $g$ is the coupling of an individual atom to the cavity mode, $\kappa_{R}$ characterizes the lossiness of the cavity coupling layer. Spectroscopically, it determines the contrast of the cavity mode in reflection, i.e., the depth of the reflectivity dip. $\Delta_{c}$ is the detuning of the incoming beam from the cavity and $\Omega$ the strength of the coupling of the beam to the cavity, $N$ is the number of atoms interacting with the $\mathrm{x}$ rays, and $\xi_{s}$ and $\delta_{\mathrm{LS}}$ are the angle-dependent 
superradiant enhancement and collective Lamb shift, respectively. This formula was derived by calculating the density matrix in the steady-state limit, having adiabatically eliminated the cavity mode. An underlying assumption is that only one resonant photon at a time enters the systemin other words, it is weakly driven by the incoming beam, which is reasonable, since the latter contains only some dozen number of photons per pulse spread over the full length of the cavity. The equation includes, however, the spectroscopic signal of the cavity, which interferes with the signal of the tantalum layers. Since the linewidth $\kappa$ is much larger than $\gamma$ (a crucial condition for the adiabatic elimination), the interference resembles the so-called Fano resonances, where a system can take two different quantum paths through a spectrally broad and a spectrally narrow state [19]. More specifically, the photon can arrive at the detector either by being absorbed by the cavity and being reemitted or by being absorbed first by the mode, then by the ensemble of resonant atoms, and then sent on its way. Omitting the cavity contributions leading to the interference in the derivation of the above formula, we get the emission signal of the resonant atoms alone (still containing cavity-induced superradiance and shifts),

$$
I=\left|\frac{N|g|^{2} \Omega}{i \gamma+\Delta+N|g|^{2}\left(i \xi_{s}+\delta_{\mathrm{LS}}\right)}\right|^{2}
$$

which we immediately see corresponds to the third term of the reflectivity in Eq. (1), i.e., the part of that term which does not stem from the cavity mode. In that sense, it is interesting to note that this purely resonant reaction still depends on the detuning of the cavity from the incoming beam, via $\xi_{s}$ and $\delta_{\mathrm{LS}}$, corresponding to what we observe. These equations are used to fit the data, with the fit results shown in Fig. 2 as well. The results for the parameters are $\kappa=283 \mathrm{eV}, \kappa_{R}=103 \mathrm{eV}, \gamma=5 \mathrm{eV}, \sqrt{N} g=41 \mathrm{eV}$. The values for the collective Lamb shift and the superradiant decay enhancement calculated from the parameters are shown in Fig. 4. The maximal collective Lamb shift is approximately $2.5 \mathrm{eV}$, the superradiant enhancement a factor of 2, leading to a total linewidth of $10 \mathrm{eV}$.

In conclusion, we have shown that $\mathrm{x}$-ray cavity quantum electrodynamics can be extended to atomic-excitonic transitions, namely the $L_{\mathrm{III}}$ transition of tantalum. Spectroscopic effects observed here include the collective Lamb shift and the observation of Fano line shapes due to interference between scattering from the cavity and the atom. We point out that the tantalum layer behave similarly to an ensemble of atoms, although the upper levels form bands. We expect that this experiment will lead to further $\mathrm{x}$ ray quantum optics work with electronic resonances, such as strong coupling between a cavity mode and an ensemble of resonant atoms. Indeed, white lines such as the tantalum $L_{\text {III }}$ resonance have a number of advantages over the nuclear resonance of ${ }^{57} \mathrm{Fe}$. For instance, the spectral width

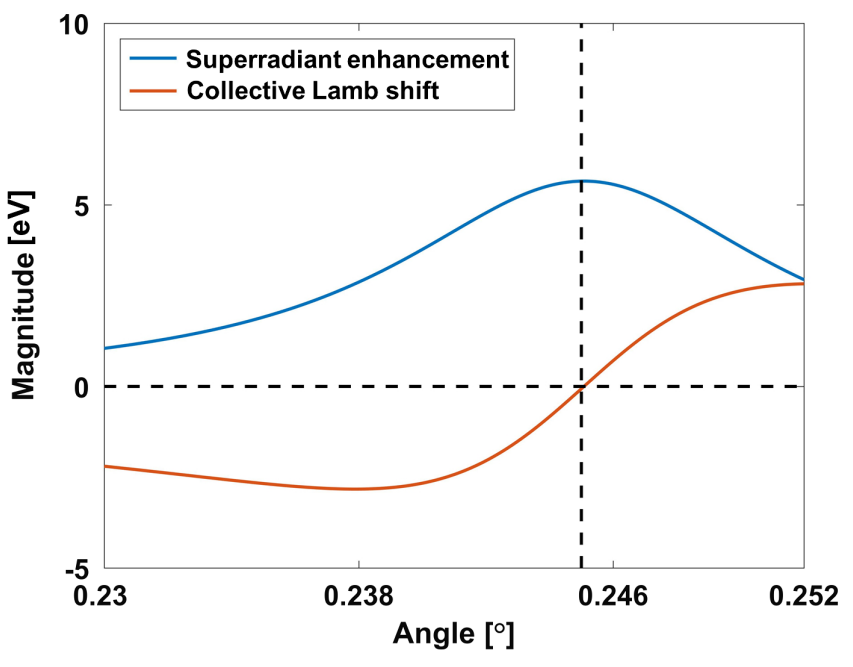

FIG. 4. The functional dependence of the superradiant decay enhancement and the collective Lamb shift of the $L_{\mathrm{III}}$-transition level. The latter changes its sign depending on the detuning, and is zero at the point of zero detuning of the cavity; the former has its maximum at that point. The influence of the cavity allows the effective "tuning" of the transition properties. The horizontal dashed line represents the position at which the effect is zero, the vertical dashed line the angular position of the cavity resonance.

of the electronic resonances is larger than that of the nuclear ones by more than 10 orders of magnitude. Hence, given the brilliance of even third generation synchrotrons and state-of-the-art X-ray free electron laser, multiphoton experiments become much easier, as the illuminating beam contains many more photons within the bandwidth of electronic resonances. This should provide new possibilities for resonance-enhanced nonlinear optics in the x-ray domain. We also point out that these results have relevance for high-precision spectroscopy in the x-ray range. The cavity mode is in principle no different from standing waves appearing in Bragg scattering or on surfaces [44], both of which could likewise influence spectroscopic signatures. In experiments in which X-ray standing waves and spectroscopy are combined [45], quantum optically induced shifts may appear and distort the results. Collective Lamb shift processes might be relevant for novel techniques involving x-ray stimulated emission [46]. Finally, the recent availability of attosecond pulses in the hard $\mathrm{x}$-ray range [47] hints at future possibilities of probing hard x-ray quantum optical effects in the temporal domain as well.

This research was carried out at the resonant scattering and diffraction beam line P09 of PETRA III at DESY. We acknowledge the support of the Helmholtz Association. This work is supported by the Cluster of Excellence "The Hamburg Centre for Ultrafast Imaging" of the Deutsche Forschungsgemeinschaft (DFG) - EXC 1074 Project No. 194651731. We thank Balaram Sahoo for early contributions to this experiment. 
*Present address: SLAC National Laboratory, 2575 Sand Hill Road, Menlo Park, California 94025, USA.

'Present address: Argonne National Laboratory, 9700 Cass Avenue, Lemont, Illinois 60439, USA.

[1] J. M. Raimond, M. Brune, and S. Haroche, Rev. Mod. Phys. 73, 565 (2001)

[2] R. E. Slusher, L. W. Hollberg, B. Yurke, J. C. Mertz, and J. F. Valley, Phys. Rev. Lett. 55, 2409 (1985).

[3] M. G. Raizen, R. J. Thompson, R. J. Brecha, H. J. Kimble, and H. J. Carmichael, Phys. Rev. Lett. 63, 240 (1989).

[4] M. Brune, F. Schmidt-Kaler, A. Maali, J. Dreyer, E. Hagley, J. M. Raimond, and S. Haroche, Phys. Rev. Lett. 76, 1800 (1996).

[5] H. Walther, B. T. H. Varcoe, B.-G. Englert, and T. Becker, Rep. Prog. Phys. 69, 1325 (2006).

[6] S. Haroche and J.-M. Raimond, Exploring the Quantum: Atoms, Cavities, and Photons (Oxford University Press, Oxford, 2006).

[7] H. Grote, K. Danzmann, K. L. Dooley, R. Schnabel, J. Slutsky, and H. Vahlbruch, Phys. Rev. Lett. 110, 181101 (2013).

[8] M. Taylor, J. Janousek, V. Daria, J. Knittel, B. Hage, H.-A. Bachor, and W. Bowen, Nat. Photonics 7, 229 (2013).

[9] J. Flick, M. Ruggenthaler, H. Appel, and A. Rubio, Proc. Natl. Acad. Sci. U.S.A. 114, 3026 (2017).

[10] T. W. Ebbesen, Acc. Chem. Res. 49, 2403 (2016).

[11] S. Smolka, W. Wuster, F. Haupt, S. Faelt, W. Wegscheider, and A. Imamoglu, Science 346, 332 (2014).

[12] M. Sidler, P. Back, O. Cotlet, A. Srivastava, T. Fink, M. Kroner, E. Demler, and A. Imamoglu, Nat. Phys. 13, 255 (2017).

[13] G. Mazza and A. Georges, Phys. Rev. Lett. 122, 017401 (2019).

[14] R. Röhlsberger, K. Schlage, B. Sahoo, S. Couet, and R. Rüffer, Science 328, 1248 (2010).

[15] R. Röhlsberger, H.-C. Wille, K. Schlage, and B. Sahoo, Nature (London) 482, 199 (2012).

[16] K. P. Heeg, H.-C. Wille, K. Schlage, T. Guryeva, D. Schumacher, I. Uschmann, K. S. Schulze, B. Marx, T. Kämpfer, G. G. Paulus, R. Röhlsberger, and J. Evers, Phys. Rev. Lett. 111, 073601 (2013).

[17] J. Haber, K. Schulze, K. Schlage, R. Loetzsch, L. Bocklage, T. Gurieva, H. Bernhardt, H.-C. Wille, R. Rüffer, I. Uschmann, G. Paulus, and R. Röhlsberger, Nat. Photonics 10, 445 (2016).

[18] J. Haber, X. Kong, C. Strohm, S. Willing, J. Gollwitzer, L. Bocklage, R. Rüffer, A. Pálffy, and R. Röhlsberger, Nat. Photonics 11, 720 (2017).

[19] K. P. Heeg, C. Ott, D. Schumacher, H.-C. Wille, R. Röhlsberger, T. Pfeifer, and J. Evers, Phys. Rev. Lett. 114, 207401 (2015).

[20] K. P. Heeg, J. Haber, D. Schumacher, L. Bocklage, H.-C. Wille, K. S. Schulze, R. Loetzsch, I. Uschmann, G. G. Paulus, R. Rüffer, R. Röhlsberger, and J. Evers, Phys. Rev. Lett. 114, 203601 (2015).

[21] T. Glover, D. Fritz, M. Cammarata, T. Allison, S. Coh, J. Feldkamp, H. Lemke, D. Zhu, Y. Feng, R. Coffee,
M. Fuchs, S. Ghimire, J. Chen, S. Shwartz, D. Reis, S. Haarris, and J. Hastings, Nature (London) 488, 603 (2012).

[22] M. Krause and J. Oliver, J. Phys. Chem. Ref. Data 8, 329 (1979).

[23] M. Brown, R. E. Peierls, and E. A. Stern, Phys. Rev. B 15, 738 (1977).

[24] F. Vagizov, V. Antonov, Y. Radeonychev, R. Shakhmuratov, and O. Kocharovskaya, Nature (London) 508, 80 (2014).

[25] K. Heeg, A. Kaldun, C. Strohm, P. Reiser, C. Ott, R. Subramanian, D. Lentdrodt, J. Haber, H.-C. Wille, S. Goerttler, R. Rüffer, C. Keitel, R. Röhlsberger, T. Pfeifer, and J. Evers, Science 357, 375 (2017).

[26] U. Fano, Phys. Rev. 124, 1866 (1961).

[27] K. P. Heeg and J. Evers, Phys. Rev. A 88, 043828 (2013).

[28] R. H. Dicke, Phys. Rev. 93, 99 (1954).

[29] M. O. Scully, Phys. Rev. Lett. 102, 143601 (2009).

[30] R. Friedberg and J. T. Manassah, Phys. Lett. A 373, 3423 (2009).

[31] A. A. Svidzinsky, J.-T. Chang, and M. O. Scully, Phys. Rev. A 81, 053821 (2010).

[32] R. Friedberg, S. Hartmann, and J. Manassah, Phys. Rep. 7, 101 (1973).

[33] M. O. Scully, Phys. Rev. Lett. 102, 143601 (2009).

[34] M. O. Scully and A. A. Svidzinsky, Science 325, 1510 (2009).

[35] A. A. Svidzinsky and M. O. Scully, Opt. Commun. 282, 2894 (2009).

[36] R. Friedberg and J. T. Manassah, Phys. Rev. A 81, 043845 (2010).

[37] A. A. Svidzinsky and M. O. Scully, Opt. Commun. 283, 753 (2010).

[38] J. T. Manassah, Adv. Opt. Photonics 4, 108 (2012).

[39] J. Ruostekoski and J. Javanainen, Phys. Rev. Lett. 117, 143602 (2016).

[40] J. Keaveney, A. Sargsyan, U. Krohn, I. G. Hughes, D. Sarkisyan, and C. S. Adams, Phys. Rev. Lett. 108, 173601 (2012).

[41] Z. Meir, O. Schwartz, E. Shahmoon, D. Oron, and R. Ozeri, Phys. Rev. Lett. 113, 193002 (2014).

[42] T. Peyrot, Y. R. P. Sortais, A. Browaeys, A. Sargsyan, D. Sarkisyan, J. Keaveney, I. G. Hughes, and C. S. Adams, Phys. Rev. Lett. 120, 243401 (2018).

[43] S. J. Roof, K. J. Kemp, M. D. Havey, and I. M. Sokolov, Phys. Rev. Lett. 117, 073003 (2016).

[44] J. Wang, M. Bedzyk, and M. Caffrey, Science 258, 775 (1992).

[45] S. Nemsak, A. Shavorskiy, O. Karslioglu, I. Zegkinoglou, A. Rattanachata, C. Conlon, A. Keqi, P. Greene, E. Burks, F. Salmassi, E. Gullikson, S.-H. Yang, K. Liu, H. Bluhm, and C. S. Fadley, Nat. Commun. 5, 5441 (2014).

[46] T. Kroll et al., Phys. Rev. Lett. 120, 133203 (2018).

[47] S. Huang, Y. Ding, Y. Feng, E. Hemsing, Z. Huang, J. Krzywinski, A. A. Lutman, A. Marinelli, T. J. Maxwell, and D. Zhu, Phys. Rev. Lett. 119, 154801 (2017). 